\title{
Oncologic PET/MRI, Part 1: Tumors of the Brain, Head and Neck, Chest, Abdomen, and Pelvis
}

\author{
Christian Buchbender ${ }^{1}$, Till A. Heusner ${ }^{1}$, Thomas C. Lauenstein ${ }^{2}$, Andreas Bockisch ${ }^{3}$, and Gerald Antoch ${ }^{1}$ \\ ${ }^{I}$ Department of Diagnostic and Interventional Radiology, University of Dusseldorf, Dusseldorf, Germany; ${ }^{2}$ Department of Diagnostic \\ and Interventional Radiology and Neuroradiology, University of Duisburg-Essen, Essen, Germany; and ${ }^{3}$ Department of Nuclear \\ Medicine, University of Duisburg-Essen, Essen, Germany
}

\begin{abstract}
Learning Objectives: On successful completion of this activity, participants should be able to describe (1) the advantages and disadvantages of PET/MRI in oncologic applications in comparison to conventional imaging methods and PET/CT; (2) the limitations of PET/MRI compared with invasive staging procedures (biopsy); and (3) the metabolic-anatomic imaging procedure of choice (PET/MRI vs. PET/CT) based on tumor entity and location.

Financial Disclosure: The authors of this article have indicated no relevant relationships that could be perceived as a real or apparent conflict of interest. CME Credit: SNM is accredited by the Accreditation Council for Continuing Medical Education (ACCME) to sponsor continuing education for physicians. SNM designates each JNM continuing education article for a maximum of 1.0 AMA PRA Category 1 Credit. Physicians should claim only credit commensurate with the extent of their participation in the activity.

For CE credit, participants can access this activity through the SNM Web site (http://www.snm.org/ce_online) through June 2013.
\end{abstract}

In oncology, staging forms the basis for prognostic consideration and directly influences patient care by determining the therapeutic approach. Cross-sectional imaging techniques, especially when combined with PET information, play an important role in cancer staging. With the recent introduction of integrated whole-body PET/MRI into clinical practice, a novel metabolic-anatomic imaging technique is now available. PET/ MRI seems to be highly accurate in T-staging of tumor entities for which MRI has traditionally been favored, such as squamous cell carcinomas of the head and neck. By adding functional MRI to PET, PET/MRI may further improve diagnostic accuracy in the differentiation of scar tissue from recurrence of tumors such as rectal cancer. This hypothesis will have to be assessed in future studies. With regard to N-staging, PET/MRI does not seem to provide a considerable benefit as compared with PET/CT but provides similar N-staging accuracy when applied as a whole-body staging approach. M-staging will benefit from MRI accuracy in the brain and the liver. The purpose of this review is to summarize the available first experiences with $\mathrm{PET} / \mathrm{MRI}$ and to outline the potential value of PET/MRI in oncologic applications for which data on PET/MRI are still lacking.

Key Words: PET/MRI; PET; MRI; cancer; oncology

J Nucl Med 2012; 53:928-938

DOI: 10.2967/jnumed.112.105338

Received Mar. 19, 2012; revision accepted May 2, 2012.

For correspondence or reprints contact: Gerald Antoch, University of Dusseldorf, Medical Faculty, Department of Diagnostic and Interventional Radiology, Moorenstrasse 5, D-40225 Dusseldorf, Germany.

E-mail: antoch@med.uni-duesseldorf.de

Published online May 11, 2012.

COPYRIGHT @ 2012 by the Society of Nuclear Medicine, Inc.
$\mathbf{I}_{\mathrm{n}}$ sideration and directly influences patient care by determining the therapeutic approach. The periodically revised standardized TNM cancer staging system (1) is pivotal for comparative treatment studies, which in an ongoing process define the most suitable therapy regime for each tumor stage and entity. Imaging plays a key role in the evaluation of local tumor extent and in the detection of potential locoregional lymph node or distant metastases. Diagnostic accuracy in the determination of the individual TNM stage, besides methodologic safety, operational availability, and cost, is the most questioned attribute when it comes to the choice of the most appropriate and accurate imaging modality for cancer staging. With the launch of integrated whole-body PET/MRI, the purpose of this review is to summarize the available first experiences with PET/MRI and to outline the potential value of PET/MRI in oncologic applications for which data on PET/MRI are still lacking. In parts of this article, we refer to our own unpublished experiences with PET/MRI that have not yet undergone a peer-review process. This contribution needs to be understood as supported solely by the authors' experience and should not be misinterpreted as evidence-based knowledge.

\section{TECHNICAL ADVANCES IN DIAGNOSTIC IMAGING}

In the past decade, the introduction of integrated metabolic-anatomic imaging with PET/CT has had a substantial influence on tumor staging and has set a new benchmark in TNM staging accuracy when compared with conventional imaging modalities (2). On the other hand, because of the lower soft-tissue contrast, PET/CT could 
not replace MRI in certain staging indications, such as T-staging of soft-tissue sarcomas, primary hepatic malignancies, and M-staging of cerebral or liver metastases (3). MRI itself has advanced significantly over the past few years and can now provide high-resolution images within a reasonable scanning time (4). In addition, several different functional MRI techniques have evolved. Among others, diffusionweighted imaging (DWI) and nuclear MR spectroscopy provide a new dimension of biologic information in MRI by enabling measurements of tissue cellularity and amino acid composition. On the PET side, detectors needed to be developed that could be operated in the presence of a high magnetic field. Avalanche photodiode detectors allow for the contemporaneous use of MRI (5), an advance that facilitated the integration of MRI and PET. This new combination was eagerly awaited to enhance the T-staging accuracy in those tumors that could not dispense with MRI and the N-staging and M-staging performance in body compartments that were superiorly depicted by PET (3). Regardless of these advances, PET/MRI has the same natural restrictions in the detection of micrometastases as other imaging modalities. Because of the technically limited spatial resolution of MRI and PET scanners, and differences in the avidity of tumors to the radionuclide, very small metastases are frequently missed when unselective radionuclides such as ${ }^{18} \mathrm{~F}-\mathrm{FDG}$ are used (6). This general limitation has to be kept in mind when the role of new imaging modalities is discussed in an oncologic context.

\section{ADVANCED MRI TECHNIQUES IN ONCOLOGY}

MRI can provide different image contrasts through adjustment of echo and repetition times, parameters that represent specific tissue characteristics. T1- and T2-weighted sequences form the basis of morphologic MRI information. In addition to mere morphologic imaging, molecular imaging is provided through a variety of functional MRI techniques. As a measure of cellularity, DWI assesses the degree to which diffusion of water molecules is restricted in the extracellular space (7). By calculation of the apparent diffusion coefficient, MR DWI, for example, increases the detection rate of metastases in normal-sized lymph nodes from $7 \%$ to $76 \%(8)$. MR spectroscopy provides a quantitative measure of the amino acid composition of a region of interest (9). These specific amino acid profiles can be used to differentiate brain tumor entities (10). Dynamic contrast-enhanced MRI allows for the calculation of different fractions of tumor perfusion, providing information on the amount of neovascularization. Quantitative dynamic contrast-enhanced MRI can be helpful for differentiating benign from malignant breast tumors and can be of use for assessing the effects of antiangiogenic tumor therapy (11). With DWI, MR spectroscopy, and dynamic contrast-enhanced MRI, we present just a selection of molecular MRI techniques valuable for oncologic applications to point out the principle that the use of MRI in integrated PET/MRI goes beyond the anatomic correlation of PET findings. The simultaneous acquisition of multiparametric MRI and PET data is awaited to create new options in molecular tumor imaging.

Our review will have 2 parts. As part 1, this article addresses tumors of the brain, head and neck, chest, abdomen, and pelvis. Part 2, to be published in a subsequent issue, reviews tumors of the bone and soft tissues, as well as melanoma and lymphoma. In both parts, distant metastases are addressed on an organ basis rather than separately for each primary tumor.

\section{TUMORS OF THE BRAIN}

\section{Cerebral Metastases}

Cerebral metastases represent the most frequent brain tumors and occur in $20 \%-40 \%$ of cancer patients. Generally, patients with cerebral metastases have a relatively short survival. Among patients with brain metastases, those with solitary or few metastases face a rather favorable prognosis. However, sensitive detection is essential to supply an appropriate potentially curative or palliative therapy to the patient. In most cases, cerebral metastases become symptomatic with headache, focal neurologic deficits, and seizures but may also be found coincidentally on staging scans. When it comes to imaging, CT and MRI are the standard modalities for the assessment of the brain. In a randomized prospective trial on preoperative staging of lung cancer patients (12), significantly smaller metastases (as small as $0.5 \mathrm{~mm}$ ) were seen on MRI than on CT. Because of the high physiologic ${ }^{18}$ F-FDG uptake of the cortex, ${ }^{18}$ F-FDG PET does not compensate for the shortcomings of CT in the detection of subcentimeter metastases. Retrospective comparative studies on ${ }^{18} \mathrm{~F}$-FDG PET and MRI reported that ${ }^{18}$ F-FDG PET detected only $61 \%$ of the metastases that were detected with MRI (13). Consequently, the diagnostic performance of integrated ${ }^{18} \mathrm{~F}$-FDG PET/CT for the detection of brain metastases was found to be weak, with a maximum sensitivity, specificity, and accuracy of $50 \%, 97 \%$, and $76 \%$ when MRI was used as the reference standard (14). On the other hand, in a prospective study ${ }^{18} \mathrm{~F}-\mathrm{FDG}$ PET was shown to be valuable for the specification of morphologically indistinguishable contrast-enhancing lesions found on MRI, because a significantly higher maximal standardized uptake value (SUV) $(4.1 \pm 1.7)$ was found in cerebral metastases than in high-grade gliomas $(1.9 \pm 0.8)$ and benign lesions $(0.6 \pm 0.3)$ (15). In addition, dual-phase ${ }^{18} \mathrm{~F}$-FDG PET is able to discriminate residual tumor from necrosis with $96 \%$ accuracy by measuring the increase in the ratio of lesion SUV to gray matter SUV (10), thus overcoming a common problem encountered in the interpretation of posttreatment MR images. Furthermore, imaging of the brain with PET is not restricted to the use of ${ }^{18} \mathrm{~F}-\mathrm{FDG}$ as a radiotracer. ${ }^{11} \mathrm{C}$-choline PET, for example, yielded significantly higher detection rates than ${ }^{18}$ F-FDG PET (23/23 [100\%] vs. $3 / 23[13 \%])$ in prospectively evaluated patients with cerebral metastases from thoracic cancer (17). 
Integrated PET/MRI of the brain was the first application available in humans (18); research using this technique focused on evaluation of primary brain tumors (19) rather than detection of metastases but established the feasibility of integrated PET/MRI of the brain. Data on the diagnostic accuracy of integrated PET/MRI for the detection of cerebral metastases hence are not available at present. Staging the brain with PET/MRI will rely mostly on the MRI component, as indicated by the presented clear advantages of MRI over PET and PET/CT (Fig. 1). However, these data were taken from studies using dedicated brain MRI protocols. A prospective study on 32 consecutive patients with solid tumors provided evidence that MRI of the brain embedded in whole-body MRI protocols detects fewer brain metastases than do dedicated brain scans (17 vs. 40) (20). Thus, the best estimate of the awaited diagnostic performance of PET/MRI in brain metastasis detection is provided by whole-body MRI studies. Prospectively comparing the diagnostic accuracy of PET/CT and whole-body MRI for M-staging demonstrated that MRI, even if not performed as a dedicated brain scan, is more accurate than ${ }^{18} \mathrm{~F}-\mathrm{FDG}$ PET/CT for the detection of cerebral metastases of different tumors (21). Whether the addition of functional MRI sequences, such as dynamic contrast-enhanced MRI and MR spectroscopy, will potentially benefit PET/MRI has not been considered so far.

\section{HEAD AND NECK SQUAMOUS CELL CARCINOMA}

\section{Initial Diagnosis and T-staging}

Although the initial diagnosis of squamous cell carcinoma (SCC) - the most important tumor entity of the head and neck area-is based on clinical and endoscopic findings, clinical inspection is regularly followed by imaging for confirmation of the suspected tumor and assessment of its extent. In recent years, PET/CT has broadly been applied for this indication and has been shown more accurate than CT alone for tumor detection and precise anatomic localization (22). The first report on integrated PET/MRI of head and neck cancer patients demonstrated superior tumor delineation (Figs. 2 and 3) and a good correlation between the metabolic ratios measured using PET/MRI and PET/CT (23). Nevertheless, reliable data on the diagnostic performance of PET/MRI to date still must be gained from studies using post hoc fusion images of MRI and PET datasets. The reported sensitivity of PET/MRI images for the detection of the primary tumor in patients with suspected head and neck SCC was 100\% according to the results of a prospective study (24), but the same study revealed that PET/MRI had little additional value compared with MRI alone, as the diagnosis was changed in only 1 of 46 patients. In cases of cervical metastases from an unknown primary tumor, the diagnostic performance of PET/MRI to date is unknown. In a report with a limited cohort of 2 patients, a clinically occult carcinoma of the tonsil was detected by fused PET/MRI but not by stand-
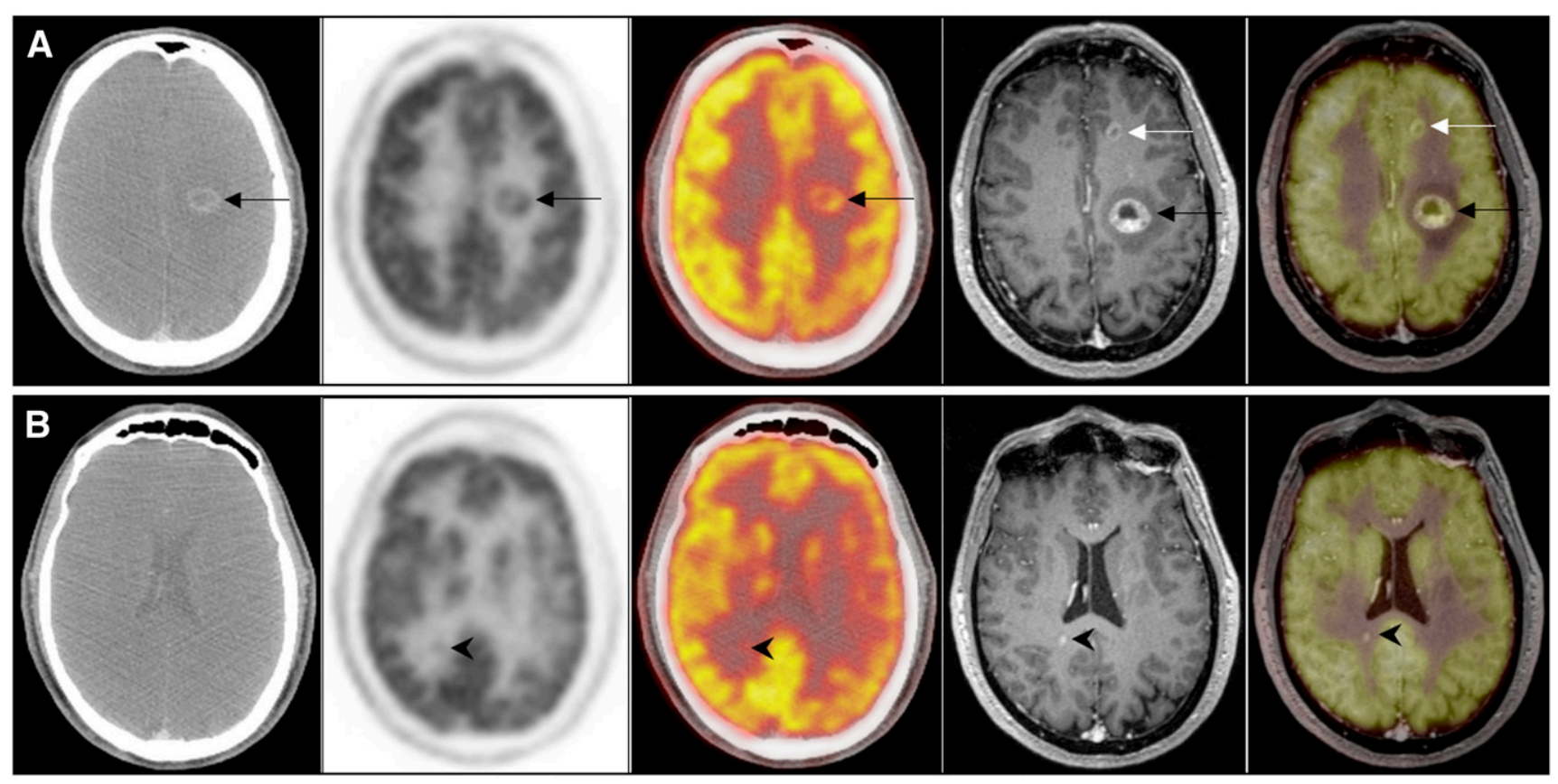

FIGURE 1. A 54-y-old patient with cerebral metastases from cancer of unknown primary. (A) Large left-hemisphere metastasis (black arrow) is visible on (from left to right) axial contrast-enhanced CT, ${ }^{18} \mathrm{~F}-\mathrm{FDG}$ PET, PET/CT, axial contrast-enhanced MRI, and PET/MRI, whereas smaller metastasis of left frontal lobe (white arrow) is visible solely on MRI and PET/MRI. Location of this metastasis directly adjacent to highly ${ }^{18} \mathrm{~F}-\mathrm{FDG}$-avid cortex leads to problems with diagnosing this lesion on ${ }^{18} \mathrm{~F}-\mathrm{FDG}$ PET scan. (B) Another subcentimetersized metastasis of right temporal lobe, clearly visible on MRI and PET/MRI of same patient (arrowhead), was only retrospectively seen as faintly increased ${ }^{18} \mathrm{~F}-\mathrm{FDG}$ activity on ${ }^{18} \mathrm{~F}-\mathrm{FDG}$ PET and PET/CT because of lack of anatomic correlate on CT. 

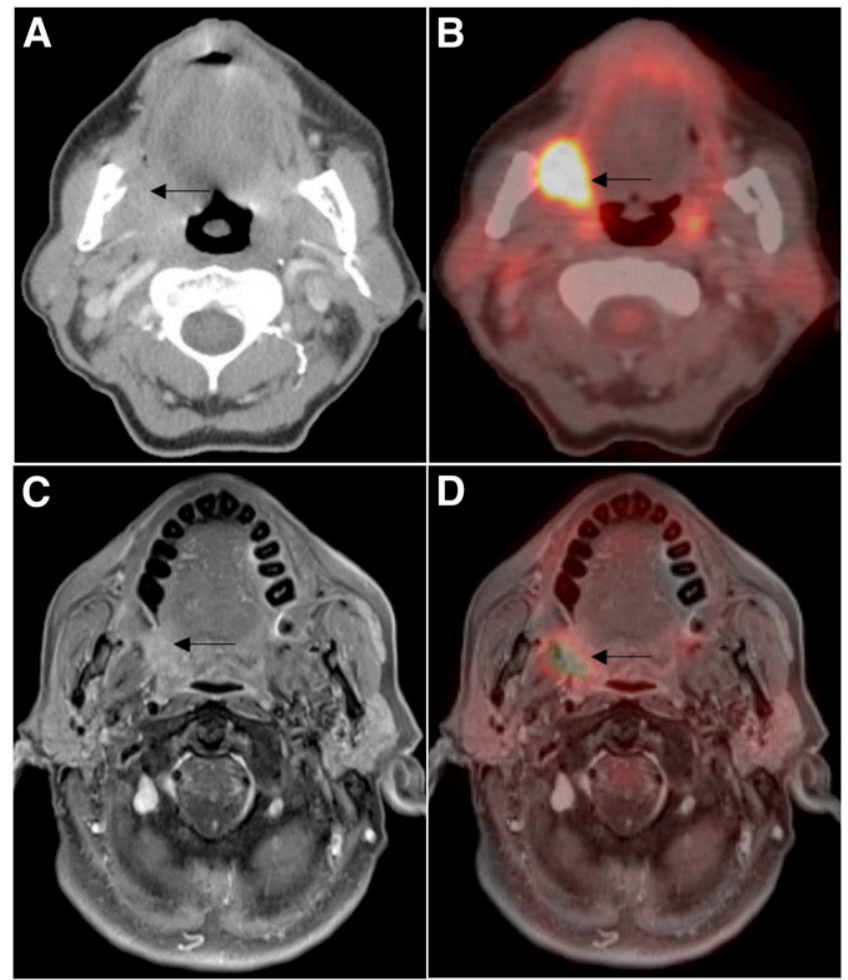

FIGURE 2. A 54-y-old man with gingival SCC arising from maxilla. Axial contrast-enhanced CT (A) shows poorly delineated soft-tissue mass (arrow) adjacent to right maxillary bone, which is clearly depicted on axial contrast-enhanced fat-suppressed T1-weighted MR image (C). ${ }^{18} \mathrm{~F}-\mathrm{FDG}$ PET/MRI (D) facilitates more precise metabolic-anatomic allocation of ${ }^{18} \mathrm{~F}-\mathrm{FDG}$-avid mass than does ${ }^{18}$ F-FDG PET/CT (B).

alone MRI (24). On the basis of the available data and initial experiences, PET/MRI in this field can be (or at least can be expected to be) as valuable as PET/CT, which has been reported to locate occult primary tumors in up to $39 \%$ of these patients (25). Considering these data from the literature, we have to acknowledge that cancer of unknown primary has not been defined consistently. If considering cancer of unknown primary as a tumor not detected even after imaging, endoscopy, and masked biopsies, the detection rate of the tumor with PET/CT goes down to $10 \%$ (in our own experience). It will be interesting to find out whether PET/MRI is able to improve this detection rate.

\section{N-Staging}

For PET/MRI and the detection of metastasis in regional lymph nodes, a sensitivity, specificity, and accuracy of $85 \%$, $92 \%$, and $89 \%$ have been reported in a prospective trial (24). PET/MRI thus exceeded the diagnostic performance found in prospective PET/CT studies-a sensitivity of $78 \%$ and a specificity of $58 \%$ for lymph node staging (26). However, PET/MRI suffers from the same weakness: a substantial number of patients $(6 / 20)$ with pathologically confirmed lymph node metastases were under-staged (24). Of these under-staged patients $(n=6), 1$ patient was falsely staged $\mathrm{N}_{0}$ instead of $\mathrm{N}_{1}$. Even if this result is considered prelim-
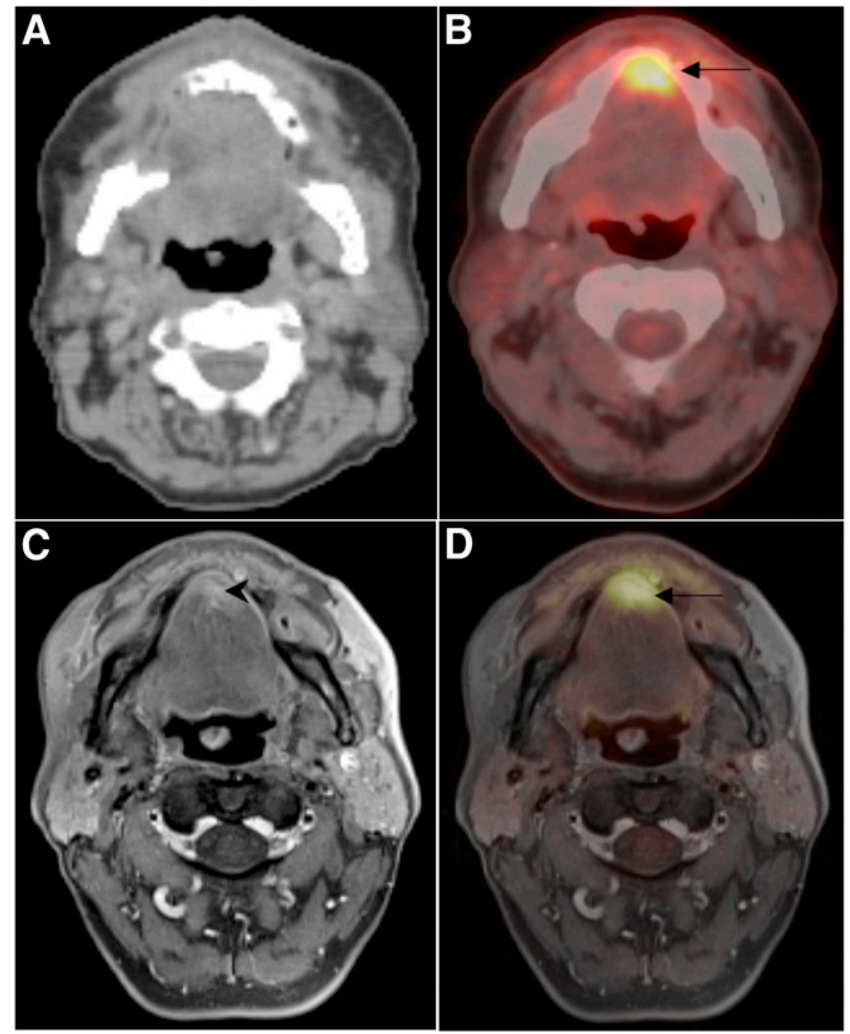

FIGURE 3. A 66-y-old patient with SCC of tongue. Axial wholebody ${ }^{18} \mathrm{~F}-\mathrm{FDG}$ PET/CT (B) shows ${ }^{18} \mathrm{~F}-\mathrm{FDG}$-avid lesion of tongue (arrow) without identifiable anatomic correlate on dedicated axial contrast-enhanced neck CT (A). Axial fat-suppressed T1-weighted MRI (C) shows contrast-enhancing mass of base of tongue (arrowhead). PET/MRI (D) shows that this tumor corresponds to ${ }^{18} \mathrm{~F}-$ FDG-avid lesion known from PET/CT.

inary, it gives note to a well-known problem in PET/CT studies. Because of the limited spatial resolution and the dependence of tumor avidity on the radionuclide in use, small metastases or micrometastases in morphologically normal lymph nodes are frequently diagnosed by histologic work-up only (6). Combining MRI with ${ }^{18}$ F-FDG PET instead of CT may enhance diagnostic performance by adding functional MRI. MR DWI is capable of the detection of SCC metastases in normal-sized $(<10$-mm short-axis diameter) lymph nodes with a sensitivity of $76 \%$ (8), which has been a tremendous improvement when compared with morphologic MRI (7\% sensitivity). Data on the combination of DWI and PET for lymph node staging in head and neck SCC are currently not available. Surgical staging by neck dissection remains the gold standard in head and neck cancer patients and most likely will not be replaced by PET/MRI

\section{Restaging and Response to Therapy}

Metabolic-anatomic cross-sectional imaging is relevant to evaluation of the therapeutic response of head and neck SCC because of the high negative predictive value (NPV), which in cases of a negative posttherapeutic scan minimizes 
the risk of local or nodal tumor recurrence (27). A recent metaanalysis comprising 2,335 head and neck SCC patients revealed a pooled sensitivity, specificity, positive predictive value (PPV), and NPV of 79.9\%, 87.5\%, 58.6\%, and 95.1\%, respectively, for posttreatment ${ }^{18} \mathrm{~F}$-FDG PET/CT of the primary tumor site. For the evaluation of nodal metastasis of the neck, these data were $72.7 \%, 87.6 \%, 52.1 \%$, and $94.5 \%$, respectively (28). A first prospective study on the value of PET/MRI in assessments for local tumor recurrence in head and neck SCC patients reported a sensitivity of $92 \%$ (24). On the basis of these first results, PET/MRI seems to improve therapy-response evaluation because of the combination of the high NPV (PET component) and the high sensitivity derived from the MRI component. Moreover, functional MRI techniques in combination with PET bear currently unused potential to further increase the performance of PET/MRI. Although the discrimination of reactively enlarged or inflammatory lymph nodes from residual or recurrent metastatic lymph nodes poses a problem that cannot be solved by PET/CT and results in false-positive findings and a low PPV of around 43\% (29), MR DWI has been reported to perform well in exactly this setting, with a sensitivity and specificity of up to $93 \%$ (30).

\section{TUMORS OF THE CHEST}

\section{Lung Tumors}

Even with modern imaging sequences, such as contrastenhanced T1 weighting with isotropic voxels, MRI is still less sensitive than CT for detection of pulmonary metastases. Prospective comparative studies on whole-body MRI and PET/CT have demonstrated the superiority of PET/CT over MRI in the detection of pulmonary metastases (139 vs. 170 pulmonary metastases) (Fig. 4) $(4,21)$. This superior

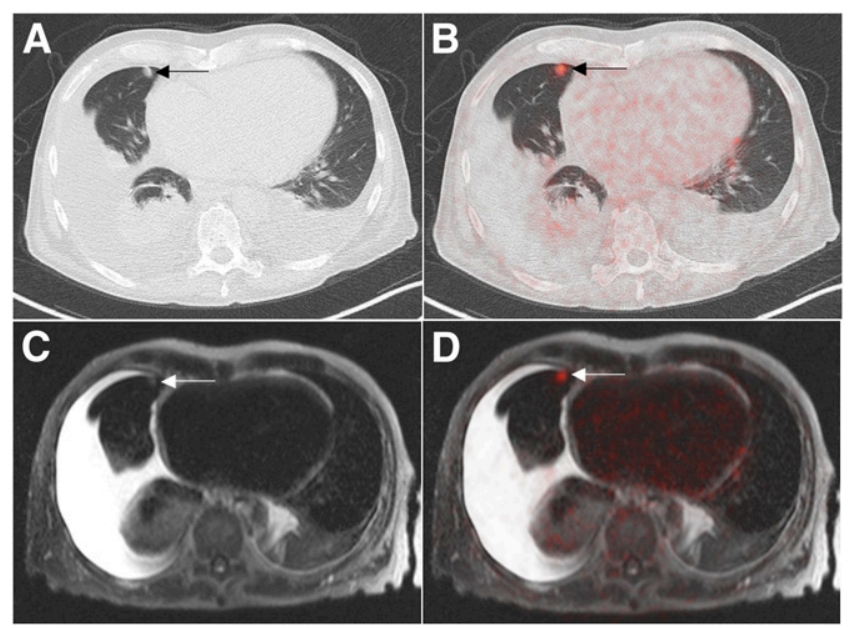

FIGURE 4. A 72-y-old patient with bronchial carcinoma of right hilum. Metastasis of right lung (arrows) found on CT (A) was confirmed by ${ }^{18} \mathrm{~F}-\mathrm{FDG}$-avid lesion on axial ${ }^{18} \mathrm{~F}-\mathrm{FDG}$ PET/CT (B). This metastasis is hardly visible on whole-body MRI using half-Fourier, single-shot, turbo spin echo sequence (C), demonstrating superior contrast of lung tissue provided by CT. PET/MRI (D) demonstrates metastasis with increased ${ }^{18} \mathrm{~F}-\mathrm{FDG}$ uptake. performance of PET/CT is based on CT accuracy rather than the PET data. On the other hand, MRI, including DWI sequences, has been shown to detect $100 \%$ of lung metastases larger than $7 \mathrm{~mm}$ found on CT (31), and the presence of small metastases below this cutoff size might be irrelevant to therapeutic decisions (32). There is also evidence that the lung-tissue contrast of MRI has the potential to be further improved by certain sequences-that is, half-Fourier, single-shot, turbo spin echo sequences (21). On the basis of the available evidence, the clinical launch of integrated PET/MRI is expected to have no benefit with regard to lung metastasis detection or primary pulmonary tumors. However, for the assessment of local tumor extent, MRI data can have benefits over CT. Local tumor infiltration into adjacent structures, such as the bronchial tree, pulmonary vessels, thoracic wall, or mediastinum, may be assessed more accurately with MRI because of its high softtissue contrast. Therefore, determination of the T-stage may be better with PET/MRI than with PET/CT. This question will, however, have to be addressed in future studies.

\section{Breast Cancer}

Initial Diagnosis and T-Staging. The awaited diagnostic performance of PET/MRI for the detection of primary breast cancer lesions and for the evaluation of local tumor extent can be deduced from several studies on softwarebased fusion of ${ }^{18} \mathrm{~F}$-FDG PET and MR mammography datasets. For the detection of primary breast carcinomas, MR mammography itself is a sensitive imaging method that uses morphologic and functional parameters but lacks specificity and PPV and is strongly dependent on the reader's degree of experience (33). In a prospective study on 36 patients, the addition of metabolic ${ }^{18}$ F-FDG PET information to MR mammography increased specificity for the detection of malignant breast lesions from 53\% to $97 \%$ (34). This increase may also be expected for simultaneous PET/ MRI of the breast. Our own group, however, did not find any statistically significant diagnostic benefit from software-based fusion of ${ }^{18} \mathrm{~F}$-FDG PET and MR mammography (35) over MR mammography alone. Equivocal lesions on MRI are typically small, and these small lesions frequently do not show increased ${ }^{18} \mathrm{~F}-\mathrm{FDG}$ uptake, even if they are malignant (in our own experience).

${ }^{18}$ F-FDG uptake has been reported to be a relevant prognostic factor in breast cancer patients, with higher SUVs indicating a poorer prognosis and being correlated with other predictors of a shorter survival such as tumor relapse, higher-grade tumors, and hormone receptor negativity (36). Hence, not only might ${ }^{18}$ F-FDG PET/MR mammography be of value for the delineation of local tumor extent, but the maximal SUV measurements might also help to estimate the prognosis of breast cancer patients. On the other hand, because of the limited sensitivity of the PET component when using ${ }^{18} \mathrm{~F}-\mathrm{FDG}$ as the radiotracer, integrated PET/ MRI will be liable to the same limitations in the detection of small breast tumors. In view of these false-negative find- 
ings with ${ }^{18} \mathrm{~F}$-FDG PET in small lesions, and other pitfalls in the use of ${ }^{18}$ F-FDG PET for the breast (e.g., false-positive lesions such as ${ }^{18} \mathrm{~F}-\mathrm{FDG}$-avid fibroadenomas), the development of joint criteria and specific reading recommendations for PET/MRI mammography seems of the utmost importance.

$N$-Staging. A recent metaanalysis on the diagnostic accuracy of MRI for the detection of axillary lymph node metastases reported a sensitivity and specificity of $90 \%$ (37). The reported sensitivity, specificity, PPV, NPV, and accuracy of ${ }^{18}$ F-FDG PET/CT for the detection of axillary lymph node metastases is $58 \%, 92 \%, 82 \%, 77 \%$, and $79 \%$, respectively (38). Both imaging modalities lead to falsenegative and false-positive results and thus cannot compete with invasive staging procedures such as sentinel lymph node biopsy and axillary lymph node dissection. These restrictions are mainly due to the lack of ability to detect micrometastases, as morphologic criteria (small axis diameter, shape, loss of a fatty hilum, central necrosis, or hypervascularization) do not apply to micrometastases, nor do micrometastases show significant ${ }^{18}$ F-FDG uptake. Because of these premises, the awaited benefit of ${ }^{18} \mathrm{~F}-\mathrm{FDG}$ PET/ MRI for the detection of axillary lymph node metastases in breast cancer patients is rather low. From the clinical point of view regarding N-stage, PET/MRI will most likely be used just as PET/CT is-as a pretest before invasive staging with the chance to avoid unnecessary axillary lymph node dissections and to triage patients to an immediate therapeutic axillary lymph node dissection if extended axillary disease is detected (38). For the detection of extraaxillary lymph node metastases and distant metastases, PET/MRI, according to our first evaluation, is as accurate as ${ }^{18}$ F-FDG PET/CT (Fig. 5), which has excellent accuracy and a direct impact on patient management (39).

Restaging and Response to Therapy. Both MRI and ${ }^{18} \mathrm{~F}-$ FDG PET are robust imaging modalities in the case of suspected breast cancer recurrence. ${ }^{18} \mathrm{~F}-\mathrm{FDG} \mathrm{PET} / \mathrm{CT}$, in a retrospective study, was proven to be an accurate modality for whole-body restaging of breast cancer patients, providing a sensitivity, specificity, PPV, NPV, and accuracy of $96 \%, 91 \%, 92 \%, 95 \%$, and 94\%, respectively (40). Especially in breast cancer patients with elevated tumor markers but negative or equivocal findings on conventional imaging, ${ }^{18}$ F-FDG PET/CT has a tremendous impact on therapeutic management (41). In view of a report that $80 \%$ of breast tumor patients have an incomplete pathologic response (42), reliable tools for the discrimination of responders from nonresponders or incomplete responders are required. In this regard, MRI is of distinct value for the prediction of pathologic complete response and the detection of residual disease, with an overall sensitivity, specificity, and accuracy of $81 \%, 93 \%$, and $84 \%$, respectively (43). The potential of MRI to assess therapeutic response is further enhanced when functional MRI techniques are added to the protocol. DWI, for example, has been shown to be sensitive to early response in a prospective study on 88 breast cancer patients undergoing neoadjuvant treatment (44). ${ }^{18} \mathrm{~F}-\mathrm{FDG}$ PET is capable of predicting therapeutic response to neoadjuvant therapy significantly earlier than conventional imaging modalities and as early as after 1 or 2 chemotherapy cycles (45). The adequate and early differentiation of responders from nonresponders has strong and direct implications toward patient management, as ineffective therapies with potentially highly toxic side effects can be stopped and alternatives can be administered. Integrated PET/MRI, joining all the benefits of morphologic and functional MRI information and metabolic PET information, is most likely to acquire an established role in the diagnostic algorithm for breast cancer patients, potentially in the settings of tumor recurrence and neoadjuvant therapy.

\section{TUMORS OF THE ABDOMEN AND PELVIS}

\section{Hepatocellular Cancer (HCC)}

Initial Diagnosis and T-staging. Data on the diagnostic performance of PET/MRI for the initial detection of hepatic primary tumors are not available. Through our evaluations of PET/CT over the past few years, we have found that ${ }^{18} \mathrm{~F}$ FDG PET/CT is of limited use in the primary diagnosis of HCC, mainly because ${ }^{18}$ F-FDG PET has limited sensitivity for well-differentiated HCCs, which tend to be non- ${ }^{18} \mathrm{~F}$ FDG-avid (46). Prospective trials have reported that for the detection of HCC, ${ }^{18} \mathrm{~F}$-FDG PET/CT has a sensitivity of around $64 \%-68 \%$ but that the sensitivity of PET may be improved by the use of radiotracers other than ${ }^{18} \mathrm{~F}-\mathrm{FDG}$
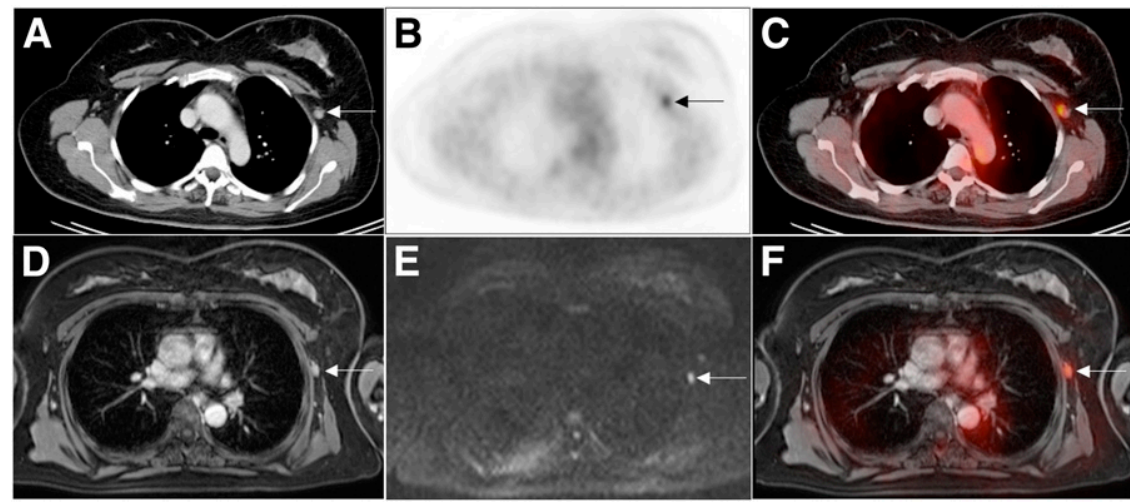

E

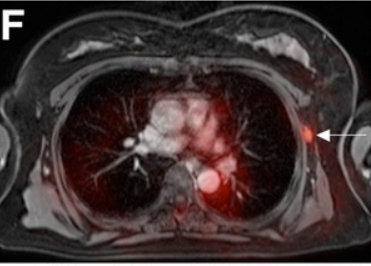

FIGURE 5. A 48-y-old patient with carcinoma of left breast. Axial contrast-enhanced CT (A), ${ }^{18} \mathrm{~F}-\mathrm{FDG}$ PET (B), and ${ }^{18} \mathrm{~F}-\mathrm{FDG}$ PET/ CT (C), and axial contrast-enhanced fatsuppressed T1-weighted MRI (D), MR DWI (E), and ${ }^{18} \mathrm{~F}-\mathrm{FDG}$ PET/MRI (F), all show lymph node metastasis of left axilla (arrows). 
(46,47). Using ${ }^{11} \mathrm{C}$-acetate (46) and ${ }^{18} \mathrm{~F}$-fluorocholine (47), the sensitivity of PET for HCC primary tumor detection was reported to rise to $84 \%$ and $88 \%$, respectively. Nonetheless, for the detection of small primary $\mathrm{HCC}(<2 \mathrm{~cm})$, the soft-tissue contrast of liver MRI is clearly superior to that of PET, especially when liver-specific contrast material such as gadolinium-ethoxybenzyl-diethylenetriamine pentaacetic acid (gadolinium-EOB-DTPA) or gadobenate acid (gadolinium-BOPTA) is applied. With liver-specific contrast material, sensitivities of around $85 \%$ have been reported in prospective studies $(48,49)$. Moreover, the additional value of functional MRI techniques in combination with PET has to be considered: MR DWI, for example, has been demonstrated to significantly improve the detection of subcentimeter-sized intrahepatic metastasis of HCC compared with conventional liver MRI alone (84\% vs. 69\%) (50). If the high sensitivity of dedicated liver MRI and the obvious potential of functional MRI can be successfully transferred to combined PET/MRI, it can be expected to perform at least as well as MRI for the primary diagnosis of HCC. The major advantage of PET/MRI scanners in the diagnostic algorithm for HCC patients is the replacement, with a single examination, of sequential MRI acquisitions for evaluation of primary tumor extent and of PET/CT for whole-body staging.

$N$-staging. Lymph node metastases occur predominantly in poorly differentiated or undifferentiated highly aggressive HCCs and thus are frequently ${ }^{18} \mathrm{~F}-\mathrm{FDG}-$ avid (51). A prospective study found that PET in combination with morphologic imaging detected more lymph node metastases than did stand-alone CT and MRI (52). Again DWI, as an example of functional MRI, can provide additional information, such as discrimination between benign and malignant abdominal lymph nodes (53).

Restaging and Response to Therapy. In the evaluation of tumor volume and viability, PET/MRI unites the metabolic information of PET, the high soft-tissue contrast of MRI, and functional MRI data. PET, by measuring tumor ${ }^{18} \mathrm{~F}-$ FDG uptake (SUV), serves as a tool for differentiation between HCCs with low biologic behavior and HCCs with highly aggressive biologic behavior. The aggressiveness of biologic behavior correlates with the volume-doubling time of HCC and thus is predictive of patient survival, with an inverse relation between SUV and survival rate (54). Furthermore, ${ }^{18} \mathrm{~F}$-FDG PET/CT has proven useful for the detection of tumor recurrence in HCC patients after liver transplantation and interventional therapy, with an overall sensitivity, specificity, and accuracy of $90 \%, 83 \%$, and $88 \%$, respectively (55). In hybrid PET/MRI scanners, dynamic contrast-enhanced sequences can add to the restaging performance. For the detection of residual viable HCC with dynamic gadolinium-enhanced MRI after transarterial chemoembolization, a sensitivity of $68 \%$, specificity of $100 \%$, and accuracy of $72 \%$ have been reported (56). However, the small number of patients in that study makes the $100 \%$ specificity questionable, and further studies are required. In a retrospective evaluation of 44 patients who had undergone hepatic tumor resection, MR DWI was shown able to differentiate histopathologic grades of HCC tumors and predict early recurrence after resection (57). These results outline the high potential of functional MRI techniques such as dynamic contrast-enhanced MRI and DWI as partners in combination with PET. The exact sensitivity and specificity of PET/MRI for tumor recurrence are currently unknown. First experiences with patients undergoing PET/ $\mathrm{CT}$ and PET/MRI after selective internal radiotherapy using ${ }^{90}$ Y-labeled particles have shown that PET/MRI improves therapeutic response assessment and early diagnosis of tumor recurrence over PET/CT (in our own experience).

\section{Liver Metastases}

Metastases to the liver are common in various malignancies and are far more frequent than primary hepatic cancers. The presence of hepatic metastases defines a high tumor stage, which is an important prognostic factor generally associated with a shorter overall survival (1). On the other hand, early and sensitive detection of liver metastases is desirable because resectioning of solitary lesions or palliative systemic and local interventional therapy for multiple hepatic metastases could prolong the patient's survival (58). Beside ultrasonography, contrastenhanced CT represents the standard diagnostic tool in liver imaging, with a sensitivity of up to $85 \%$ for the detection of metastases (59). Liver MRI with hepatobiliary contrast agents (gadolinium-EOB-DTPA or gadolinium-BOPTA) is the most accurate currently available imaging modality for the detection of small hepatic metastases (60). With a sensitivity, specificity, NPV, and PPV of $100 \%, 71 \%, 97 \%$, and 100\%, MRI outperforms ${ }^{18}$ F-FDG PET/CT (93\%, 71\%, 97\%, and 57\%) (60). ${ }^{18}$ F-FDG PET has been shown to be valuable in the response assessment of liver metastases undergoing systemic or local interventional therapy. ${ }^{18} \mathrm{~F}-\mathrm{FDG}$ PET can solve the problem of distinguishing between a marginal zone of reactive hyperperfusion, which is frequently found at the rim of metastases on contrast-enhanced CT after radiofrequency ablation, and residual viable tumor tissue (61) and hence is superior to CT in radiofrequency ablation response assessment (65\% vs. 44\%) (62). Significantly reduced SUVs have been found in liver metastases from colorectal carcinoma with a pathologically confirmed response to systemic therapy, compared with nonresponsive metastases (63). Metastases treated by radiofrequency ablation that were found to be ${ }^{18}$ F-FDG PET-negative within $3 \mathrm{wk}$ after treatment were less likely to relapse; in contrast, most persistently ${ }^{18}$ F-FDG PET-positive metastases recurred within the follow-up time of 16 mo (64). Recently, a change in maximal SUV between the preinterventional stage and 3 mo after selective internal radiation therapy of liver metastases from breast cancer was identified as the only independent predictive factor for patient survival (65). In this regard, functional MRI sequences and PET could provide complementary information on the viability of tumor tissue after therapy. 
A study by Donati et al. on the diagnostic performance of PET/MRI compared fused ${ }^{18} \mathrm{~F}-\mathrm{FDG}$ PET and gadoliniumEOB-DTPA-enhanced MRI retrospectively versus standalone gadolinium-EOB-DTPA-enhanced MRI and integrated PET/CT for liver lesion detection (66). In that study, PET/ MRI, with a sensitivity and specificity of up to $93 \%$ and $96 \%$, respectively, was significantly more accurate than $\mathrm{PET} / \mathrm{CT}$ ( $76 \%$ and $85 \%$, respectively). The evaluating radiologists rated PET/MRI as providing significantly greater confidence for discrimination between benign and malignant liver lesions. For the detection of hepatic lesions larger than $1 \mathrm{~cm}$, PET/MRI had an area under the receiver-operatingcharacteristic curve of up to 0.96 , representing a perfect test of this question. Compared with stand-alone MRI, PET/MRI led to a nonsignificant increase in sensitivity from $91 \%$ to $93 \%$, with a slightly lower specificity of $96 \%$ for PET/MRI versus $100 \%$ for MRI alone. Interestingly, both gadoliniumEOB-DTPA-enhanced MRI and PET/MRI performed better than PET/CT for the detection and characterization of lesions smaller than $1 \mathrm{~cm}(66)$. Another study, on the diagnostic accuracy of retrospectively fused PET/MR images, was performed on patients who had neuroendocrine tumors with suspected liver metastases (67). In that study, ${ }^{68} \mathrm{Ga}$ DOTATOC, a radiolabeled somatostatin analog, was used for PET (68). Fusion ${ }^{68}$ Ga-DOTATOC and gadoliniumEOB-DTPA PET/MRI for the detection of hepatic neuroendocrine tumor metastases had a sensitivity, specificity, NPV, and PPV of 91\%, 96\%, 87\%, and 97\%, respectively, and compared with PET/CT (74\%, 88\%, 69\%, and 93\%, respectively) was significantly more accurate and slightly but not significantly more accurate than stand-alone MRI $(88 \%, 87 \%, 83 \%$, and $93 \%$, respectively). A subanalysis on the influence of tumor grade revealed that stand-alone ${ }^{68}$ Ga-DOTATOC PET had a low sensitivity (5\%) for metastases of poorly differentiated neuroendocrine tumors. In these tumors, the additional MRI information increased lesion detection over PET alone (67). In summary, both presented studies on PET/MRI for the detection of hepatic metastases attested a high accuracy for this technique that exceeded the performance of PET/CT (Fig. 6). It seems that MRI better compensates for the drawbacks in PET, especially in small hepatic lesions (67), and enhances reader confidence in lesion discrimination (66). However, PET/ MRI does not seem to have any benefits over MRI alone.

\section{Colorectal Cancer}

Initial Diagnosis and T-Staging. Software-based PET/ MR images have been reported to provide no significant additional value in the preoperative staging of patients with rectal cancer, compared with pelvic MRI in conjunction with abdominal CT and chest radiography (69). The MRI component detected $100 \%$ of all rectal tumors $(n=23)$ but was inaccurate for the determination of tumor stage, as 11 of 23 patients were over-staged. The sensitivity and specificity of MRI for the T category of rectal cancer is $87 \%$ and $75 \%$, respectively, based on the results of a recent meta-

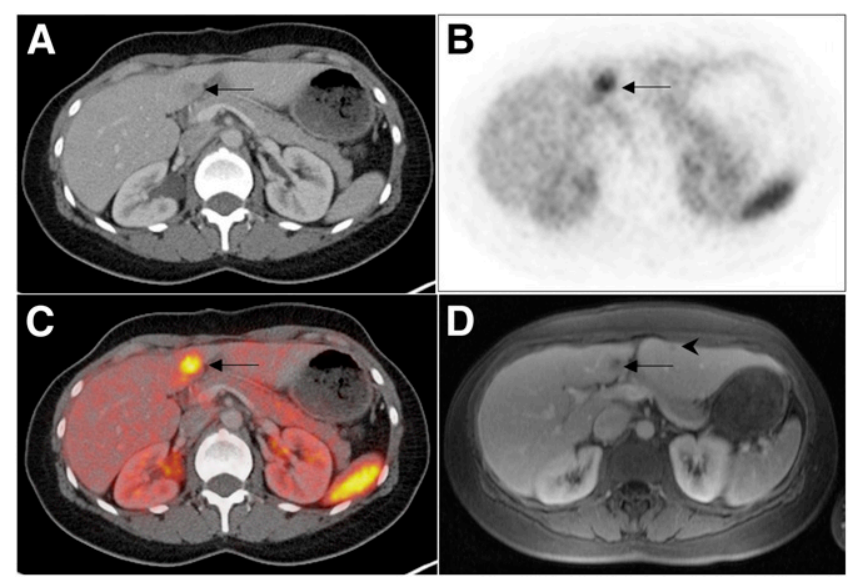

FIGURE 6. A 51-y-old patient with neuroendocrine tumor of small intestine. Axial contrast-enhanced CT (A) depicts metastasis to liver segment IV (arrow), which is strongly ${ }^{68} \mathrm{Ga}$-DOTATOC-avid on PET (B) and PET/CT (C). (D) Axial contrast-enhanced fat-suppressed T1weighted MRI shows additional hypointense lesion in liver segment III (arrowhead) that was confirmed to be metastasis on follow-up.

analysis (70). Besides endorectal ultrasonography, MRI is 1 of the 2 currently favorable preoperative imaging methods for assessment of the bowel wall and potential mesorectal infiltration (71) (Table 1). ${ }^{18} \mathrm{~F}-\mathrm{FDG}$ PET cannot relevantly add to the accuracy of T-staging, as we learned from the application of ${ }^{18} \mathrm{~F}$-FDG PET or ${ }^{18} \mathrm{~F}-\mathrm{FDG}$ PET/CT to wholebody staging in colorectal cancer patients over the past few years (72). Despite the lack of reliable data, we assume that PET/MRI will not be better than MRI alone in the assessment of local tumor extent in colorectal cancer patients.

$\mathrm{N}$-Staging. The reported sensitivity of PET/MRI images for the detection of lymph node metastases in preoperative rectal carcinoma patients is only $44 \%$ (69). In the same retrospective analysis, a specificity and PPV of $100 \%$ and a NPV of $74 \%$ were reported. The authors reported that PET was never positive in the absence of positive lymph nodes on MRI; the pathologically determined size of metastases within lymph nodes rated positively on MRI ranged from 8 to $17 \mathrm{~mm}$ in that study (69). In a metaanalysis on the diagnostic performance of MRI for the detection of lymph node metastases, a sensitivity of up to $77 \%$ and a specificity of $71 \%$ were reported (70). Interestingly, the overall diagnostic accuracy for lymph node metastasis detection could be raised to $90 \%$ if ${ }^{18} \mathrm{~F}-\mathrm{FDG}$ PET/ $\mathrm{CT}$ and MRI were combined; the sensitivity, specificity, PPV, and NPV of combined MRI and ${ }^{18} \mathrm{~F}-\mathrm{FDG}$ PET/CT were $94 \%$, $83 \%, 89 \%, 91 \%$, respectively (73). These initial experiences with PET/MRI reflect the general problem of lymph node micrometastasis detection with any imaging modality.

Restaging and Response to Therapy. PET/MRI combines the advantages of MRI, functional MRI, and ${ }^{18} \mathrm{~F}-\mathrm{FDG}$ PET for the assessment of therapeutic response and tumor relapse. MRI provides relevant prognostic information on colorectal cancer patients. In a retrospective evaluation, the tumor-free margin on preoperative MRI has been shown to be predictive of tumor recurrence and patient survival (74). 
TABLE 1

Indications in Which PET/MRI May Be Favorable over PET/CT, Depending on Tumor Entity

\begin{tabular}{|c|c|c|c|c|c|c|}
\hline \multirow[b]{2}{*}{ Tumor entity } & \multicolumn{4}{|c|}{$\begin{array}{c}\text { Most frequent site of } \\
\text { metastases }^{*}\end{array}$} & \multicolumn{2}{|c|}{$\mathrm{PET} / \mathrm{MRI}$ is favorable over PET/CT for... } \\
\hline & Brain & Lung & Liver & Bone & Staging category ${ }^{\dagger}$ & Special objective/prognostic factor \\
\hline Head and neck SCC & - & + & - & + & $\mathrm{T}$ & Extracapsular spread; bone infiltration \\
\hline Non-small cell lung cancer & + & + & + & + & $\mathrm{M}$ & Distant metastases \\
\hline Breast cancer & + & + & + & + & $\mathrm{T} / \mathrm{M}$ & $\begin{array}{l}\text { Primary diagnosis and T-stage } \\
\text { (benefit compared with PET/CT; } \\
\text { potential benefit over MRI mammography } \\
\text { alone is questionable); distant metastases }\end{array}$ \\
\hline $\mathrm{HCC}$ & - & + & + & + & $\mathrm{T}$ & Pretransplantation evaluation \\
\hline Colorectal carcinoma & - & + & + & - & $\mathrm{T} / \mathrm{M}$ & $\begin{array}{l}\text { Circumferential resection margin; } \\
\text { liver metastases; tumor regression rate } \\
\text { to neoadjuvant therapy }\end{array}$ \\
\hline
\end{tabular}

${ }^{*}$ Frequency of metastatic spread (frequently [+], rare [-]) according to AJCC Cancer Staging Manual, seventh edition.

${ }^{\dagger} \mathrm{PET} / \mathrm{CT}$ and PET/MRI are considered equally accurate for $\mathrm{N}$-staging, and thus importance of $\mathrm{N}$-staging is not accounted for.

For the assessment of this tumor-free distance to the mesorectal fascia, a recent metaanalysis showed MRI to be reliable, with a sensitivity and specificity of $77 \%$ and $94 \%$, respectively (70). In locally advanced rectal cancer, a combination of morphologic and MR DWI could also predict the tumor clearance of the mesorectal fascia yielded by neoadjuvant radiochemotherapy (75). Furthermore, MR DWI has been reported to provide an imaging biomarker for tumor invasiveness. Lower apparent diffusion coefficient values correlated significantly with more aggressive tumor profiles, including high grades, high frequency of lymph node metastases, and invasion of the mesorectal fascia (76).

In patients with clinically suspected local recurrence, sensitivity ranged from $84 \%$ to $100 \%$ and specificity from $74 \%$ to $83 \%$ for conventional MRI, and an increase of diagnostic performance by adding functional MRI information (DWI) has been reported (77). Because of local changes after surgery or radiochemotherapy such as scar tissue or desmoplastic reactions, residual or locally recurrent tumors can be difficult to identify on the basis of morphologic criteria (78). In this setting, one would consider additional metabolic information desirable for a correct restaging. Tissue regeneration and inflammation, however, lead to increased ${ }^{18} \mathrm{~F}$-FDG uptake on ${ }^{18} \mathrm{~F}$-FDG PET. Because of this limitation, ${ }^{18} \mathrm{~F}$-FDG PET/CT was not able to predict histopathologic tumor response after radiochemotherapy when scanning was performed shortly after therapy (79). The reported sensitivity, specificity, accuracy, PPV, and NPV of ${ }^{18} \mathrm{~F}-\mathrm{FDG}$ PET/CT for the detection of local colorectal cancer recurrence were $84 \%, 88 \%, 87 \%, 76 \%$, and $92 \%$, respectively (80). In patients with suspected nonlocal recurrence, whole-body imaging is favorable for a complete restaging. PET/MRI integrates the advantages of MRI and ${ }^{18} \mathrm{~F}-\mathrm{FDG}$ PET and thus may evolve as the first-line restaging modality in colorectal cancer patients with suspected tumor relapse or newly developed metastases.

\section{CONCLUSION}

Literature on truly integrated PET/MRI in oncologic applications is limited. The first experiences with this imaging technique report what may have been expected when the available data on whole-body MRI and PET/CT in oncology were considered. PET/MRI seems to be highly accurate in T-staging of those tumor entities for which MRI has traditionally been favored, such as SCCs of the head and neck. Thus, coupling PET with MRI will be clinically relevant in cases in which the soft-tissue contrast of MRI outperforms that of CT. With regard to N-staging, PET/ MRI does not seem to provide a considerable benefit as compared with PET/CT but provides similar N-staging accuracy when applied as a whole-body staging approach. M-staging will benefit from MRI accuracy in the brain and the liver. The currently available literature focuses on ${ }^{18} \mathrm{~F}-$ FDG-based PET investigations. Specific radiotracers will have to be addressed in the future. Furthermore, the use of morphologic MRI techniques for the mere anatomic correlation of PET findings seems unlikely to tap the full potential of integrated PET/MRI. The true value of this new modality rather lies in the simultaneous acquisition of functional MRI parameters (e.g., DWI, dynamic contrast-enhanced MRI, and MR spectroscopy) and metabolic PET information. For this reason, the development of diseaseand organ-specific PET/MRI protocols is a focus of the ongoing process of implementing this technique clinically. In summary, oncologic indications for PET/MRI will be defined by the soft-tissue contrast of MRI. All other indications will probably remain the domain of PET/CT.

\section{REFERENCES}

1. Edge SB, Byrd DR, Compton CC, et al., eds. AJCC Cancer Staging Handbook: From the AJCC Cancer Staging Manual. 7th ed. New York, NY: Springer; 2009.

2. Antoch G, Saoudi N, Kuehl H, et al. Accuracy of whole-body dual-modality fluorine-18-2-fluoro-2-deoxy-D-glucose positron emission tomography and 
computed tomography (FDG-PET/CT) for tumor staging in solid tumors: comparison with CT and PET. J Clin Oncol. 2004;22:4357-4368.

3. Antoch G, Bockisch A. Combined PET/MRI: a new dimension in whole-body oncology imaging? Eur J Nucl Med Mol Imaging. 2009;36(suppl 1):S113-S120.

4. Antoch G, Vogt FM, Freudenberg LS, et al. Whole-body dual-modality PET/CT and whole-body MRI for tumor staging in oncology. JAMA. 2003;290:31993206.

5. Melcher CL. Scintillation crystals for PET. J Nucl Med. 2000;41:1051-1055.

6. Stoeckli SJ, Steinert H, Pfaltz M, Schmid S. Is there a role for positron emission tomography with ${ }^{18} \mathrm{~F}$-fluorodeoxyglucose in the initial staging of nodal negative oral and oropharyngeal squamous cell carcinoma. Head Neck. 2002;24:345-349.

7. Zhong JH, Gore JC. Studies of restricted diffusion in heterogeneous media containing variations in susceptibility. Magn Reson Med. 1991;19:276-284.

8. Vandecaveye V, De Keyzer F, Vander Poorten V, et al. Head and neck squamous cell carcinoma: value of diffusion-weighted MR imaging for nodal staging. Radiology. 2009;251:134-146.

9. Morita N, Harada M, Otsuka H, Melhem ER, Nishitani H. Clinical application of MR spectroscopy and imaging of brain tumor. Magn Reson Med Sci. 2010;9: $167-175$.

10. Poptani H, Gupta RK, Roy R, Pandey R, Jain VK, Chhabra DK. Characterization of intracranial mass lesions with in vivo proton MR spectroscopy. AJNR. 1995; $16: 1593-1603$.

11. Gore JC, Manning HC, Quarles CC, Waddell KW, Yankeelov TE. Magnetic resonance in the era of molecular imaging of cancer. Magn Reson Imaging. 2011;29:587-600

12. Yokoi K, Kamiya N, Matsuguma H, et al. Detection of brain metastasis in potentially operable non-small cell lung cancer: a comparison of CT and MRI. Chest. 1999;115:714-719.

13. Rohren EM, Provenzale JM, Barboriak DP, Coleman RE. Screening for cerebral metastases with FDG PET in patients undergoing whole-body staging of noncentral nervous system malignancy. Radiology. 2003;226:181-187.

14. Krüger S, Mottaghy FM, Buck AK, et al. Brain metastasis in lung cancer: comparison of cerebral MRI and ${ }^{18} \mathrm{~F}-\mathrm{FDG}$-PET/CT for diagnosis in the initial staging. Nuklearmedizin. 2011;50:101-106.

15. Kwee SA, Ko JP, Jiang CS, Watters MR, Coel MN. Solitary brain lesions enhancing at MR imaging: evaluation with fluorine 18 fluorocholine PET. Radiology. 2007;244:557-565.

16. Horky LL, Hsiao EM, Weiss SE, Drappatz J, Gerbaudo VH. Dual phase FDGPET imaging of brain metastases provides superior assessment of recurrence versus post-treatment necrosis. J Neurooncol. 2011;103:137-146.

17. Pieterman RM, Que TH, Elsinga $\mathrm{PH}$, et al. Comparison of ${ }^{11} \mathrm{C}$-choline and ${ }^{18} \mathrm{~F}-\mathrm{FDG}$ PET in primary diagnosis and staging of patients with thoracic cancer. J Nucl Med. 2002;43:167-172.

18. Schlemmer HP, Pichler BJ, Schmand M, et al. Simultaneous MR/PET imaging of the human brain: feasibility study. Radiology. 2008;248:1028-1035.

19. Boss A, Bisdas S, Kolb A, et al. Hybrid PET/MRI of intracranial masses: initial experiences and comparison to PET/CT. J Nucl Med. 2010;51:1198-1205.

20. Thomson V, Pialat JB, Gay F, et al. Whole-body MRI for metastases screening: a preliminary study using 3D VIBE sequences with automatic subtraction between noncontrast and contrast enhanced images. Am J Clin Oncol. 2008;31:285-292.

21. Schmidt GP, Baur-Melnyk A, Herzog P, et al. High-resolution whole-body magnetic resonance image tumor staging with the use of parallel imaging versus dual-modality positron emission tomography-computed tomography: experience on a 32-channel system. Invest Radiol. 2005;40:743-753.

22. Veit-Haibach P, Luczak C, Wanke I, et al. TNM staging with FDG-PET/CT in patients with primary head and neck cancer. Eur J Nucl Med Mol Imaging. 2007;34:1953-1962.

23. Boss A, Stegger L, Bisdas S, et al. Feasibility of simultaneous PET/MR imaging in the head and upper neck area. Eur Radiol. 2011;21:1439-1446.

24. Nakamoto Y, Tamai K, Saga T, et al. Clinical value of image fusion from MR and PET in patients with head and neck cancer. Mol Imaging Biol. 2009;11:46-53.

25. Wong WL, Sonoda LI, Gharpurhy A, et al. ${ }^{18} \mathrm{~F}$-fluorodeoxyglucose positron emission tomography/computed tomography in the assessment of occult primary head and neck cancers: an audit and review of published studies. Clin Oncol (R Coll Radiol). 2012;24:190-195.

26. Liao CT, Wang HM, Huang SF, et al. PET and PET/CT of the neck lymph nodes improves risk prediction in patients with squamous cell carcinoma of the oral cavity. J Nucl Med. 2011;52:180-187.

27. Ong SC, Schöder H, Lee NY, et al. Clinical utility of ${ }^{18} \mathrm{~F}-\mathrm{FDG}$ PET/CT in assessing the neck after concurrent chemoradiotherapy for locoregional advanced head and neck cancer. J Nucl Med. 2008;49:532-540.

28. Gupta T, Master Z, Kannan S, et al. Diagnostic performance of post-treatment FDG PET or FDG PET/CT imaging in head and neck cancer: a systematic review and meta-analysis. Eur J Nucl Med Mol Imaging. 2011;38:2083-2095.
29. Yao M, Smith RB, Graham MM, et al. The role of FDG PET in management of neck metastasis from head-and-neck cancer after definitive radiation treatment. Int J Radiat Oncol Biol Phys. 2005;63:991-999.

30. Razek AA, Megahed AS, Denewer A, Motamed A, Tawfik A, Nada N. Role of diffusion-weighted magnetic resonance imaging in differentiation between the viable and necrotic parts of head and neck tumors. Acta Radiol. 2008;49:364370.

31. Liu J, Yang X, Li F, Wang X, Jiang X. Preliminary study of whole-body diffusionweighted imaging in detecting pulmonary metastatic lesions from clear cell renal cell carcinoma: comparison with CT. Acta Radiol. 2011;52:954-963.

32. Lauenstein TC, Goehde SC, Herborn CU, et al. Whole-body MR imaging: evaluation of patients for metastases. Radiology. 2004;233:139-148.

33. Kuhl C. The current status of breast MR imaging. Part 1. Choice of technique, image interpretation, diagnostic accuracy, and transfer to clinical practice. $R a$ diology. 2007;244:356-378.

34. Moy L, Noz ME, Maguire GQ Jr, et al. Role of fusion of prone FDG-PET and magnetic resonance imaging of the breasts in the evaluation of breast cancer. Breast J. 2010;16:369-376.

35. Hahn S, Heusner T, Kummel S, et al. Comparison of FDG-PET/CT and bone scintigraphy for detection of bone metastases in breast cancer. Acta Radiol. 2011;52:1009-1014.

36. Uematsu T, Kasami M, Yuen S. Comparison of FDG PET and MRI for evaluating the tumor extent of breast cancer and the impact of FDG PET on the systemic staging and prognosis of patients who are candidates for breast-conserving therapy. Breast Cancer. 2009;16:97-104.

37. Harnan SE, Cooper KL, Meng Y, et al. Magnetic resonance for assessment of axillary lymph node status in early breast cancer: a systematic review and metaanalysis. Eur J Surg Oncol. 2011;37:928-936.

38. Heusner TA, Kuemmel S, Hahn S, et al. Diagnostic value of full-dose FDG PET/ CT for axillary lymph node staging in breast cancer patients. Eur J Nucl Med Mol Imaging. 2009;36:1543-1550.

39. Aukema TS, Straver ME, Peeters MJ, et al. Detection of extra-axillary lymph node involvement with FDG PET/CT in patients with stage II-III breast cancer. Eur J Cancer. 2010;46:3205-3210.

40. Murakami R, Kumita SI, Yoshida T, et al. FDG-PET/CT in the diagnosis of recurrent breast cancer. Acta Radiol. 2012;53:12-16.

41. Filippi V, Malamitsi J, Vlachou F, et al. The impact of FDG-PET/CT on the management of breast cancer patients with elevated tumor markers and negative or equivocal conventional imaging modalities. Nucl Med Commun. 2011;32:8590.

42. Avril N, Sassen S, Roylance R. Response to therapy in breast cancer. J Nucl Med. 2009;50(suppl 1):55S-63S.

43. Chen JH, Bahri S, Mehta RS, et al. Breast cancer: evaluation of response to neoadjuvant chemotherapy with 3.0-T MR imaging. Radiology. 2011;261:735743.

44. Wang XH, Peng WJ, Tan HN, et al. Value of diffusion weighted imaging (DWI) in evaluating early response to neoadjuvant chemotherapy in locally advanced breast cancer [in Chinese]. Zhonghua Zhong Liu Za Zhi. 2010;32:377-381.

45. Ueda S, Tsuda H, Saeki T, et al. Early reduction in standardized uptake value after one cycle of neoadjuvant chemotherapy measured by sequential FDG PET/ $\mathrm{CT}$ is an independent predictor of pathological response of primary breast cancer. Breast J. 2010;16:660-662.

46. Park JW, Kim JH, Kim SK, et al. A prospective evaluation of ${ }^{18} \mathrm{~F}-\mathrm{FDG}$ and ${ }^{11} \mathrm{C}$ acetate PET/CT for detection of primary and metastatic hepatocellular carcinoma. J Nucl Med. 2008;49:1912-1921.

47. Talbot JN, Fartoux L, Balogova S, et al. Detection of hepatocellular carcinoma with PET/CT: a prospective comparison of ${ }^{18} \mathrm{~F}$-fluorocholine and ${ }^{18} \mathrm{~F}-\mathrm{FDG}$ in patients with cirrhosis or chronic liver disease. J Nucl Med. 2010;51:1699-1706.

48. Di Martino M, Marin D, Guerrisi A, et al. Intraindividual comparison of gadoxetate disodium-enhanced MR imaging and 64-section multidetector CT in the detection of hepatocellular carcinoma in patients with cirrhosis. Radiology. 2010;256:806-816.

49. Park G, Kim YK, Kim CS, Yu HC, Hwang SB. Diagnostic efficacy of gadoxetic acid-enhanced MRI in the detection of hepatocellular carcinomas: comparison with gadopentetate dimeglumine. Br J Radiol. 2010;83:1010-1016.

50. Yu JS, Chung JJ, Kim JH, et al. Detection of small intrahepatic metastases of hepatocellular carcinomas using diffusion-weighted imaging: comparison with conventional dynamic MRI. Magn Reson Imaging. 2011;29:985-992.

51. Torizuka $\mathrm{T}$, Tamaki N, Inokuma $\mathrm{T}$, et al. In vivo assessment of glucose metabolism in hepatocellular carcinoma with FDG-PET. J Nucl Med. 1995;36: 1811-1817.

52. Yoon KT, Kim JK, Kim Do Y, et al. Role of ${ }^{18} \mathrm{~F}$-fluorodeoxyglucose positron emission tomography in detecting extrahepatic metastasis in pretreatment staging of hepatocellular carcinoma. Oncology. 2007;72(suppl 1):104-110. 
53. Akduman EI, Momtahen AJ, Balci NC, Mahajann N, Havlioglu N, Wolverson MK. Comparison between malignant and benign abdominal lymph nodes on diffusion-weighted imaging. Acad Radiol. 2008;15:641-646.

54. Shiomi S, Nishiguchi S, Ishizu H, et al. Usefulness of positron emission tomography with fluorine-18-fluorodeoxyglucose for predicting outcome in patients with hepatocellular carcinoma. Am J Gastroenterol. 2001;96:1877-1880.

55. Sun L, Guan YS, Pan WM, et al. Metabolic restaging of hepatocellular carcinoma using whole-body F-FDG PET/CT. World J Hepatol. 2009;1:90-97.

56. Bolog N, Pfammatter T, Müllhaupt B, Andreisek G, Weishaupt D. Double-contrast magnetic resonance imaging of hepatocellular carcinoma after transarterial chemoembolization. Abdom Imaging. 2008;33:313-323.

57. Nakanishi M, Chuma M, Hige S, et al. Relationship between diffusion-weighted magnetic resonance imaging and histological tumor grading of hepatocellular carcinoma. Ann Surg Oncol. 2012;19:1302-1309.

58. Pathak S, Jones R, Tang JM, et al. Ablative therapies for colorectal liver metastases: a systematic review. Colorectal Dis. 2011;13:e252-e265.

59. Piscaglia F, Corradi F, Mancini M, et al. Real time contrast enhanced ultrasonography in detection of liver metastases from gastrointestinal cancer. BMC Cancer. 2007;7:171.

60. Seo HJ, Kim MJ, Lee JD, Chung WS, Kim YE. Gadoxetate disodium-enhanced magnetic resonance imaging versus contrast-enhanced ${ }^{18} \mathrm{~F}$-fluorodeoxyglucose positron emission tomography/computed tomography for the detection of colorectal liver metastases. Invest Radiol. 2011;46:548-555.

61. Antoch G, Vogt FM, Veit P, et al. Assessment of liver tissue after radiofrequency ablation: findings with different imaging procedures. J Nucl Med. 2005;46:520525.

62. Veit P, Antoch G, Stergar H, Bockisch A, Forsting M, Kuehl H. Detection of residual tumor after radiofrequency ablation of liver metastasis with dual-modality PET/CT: initial results. Eur Radiol. 2006;16:80-87.

63. Findlay M, Young H, Cunningham D, et al. Noninvasive monitoring of tumor metabolism using fluorodeoxyglucose and positron emission tomography in colorectal cancer liver metastases: correlation with tumor response to fluorouracil. J Clin Oncol. 1996;14:700-708.

64. Choi J. Imaging of hepatic metastases. Cancer Control. 2006;13:6-12.

65. Haug AR, Tiega Donfack BP, Trumm C, et al. ${ }^{18}$ F-FDG PET/CT predicts survival after radioembolization of hepatic metastases from breast cancer. $\mathrm{J} \mathrm{Nucl}$ Med. 2012;53:371-377.

66. Donati OF, Hany TF, Reiner CS, et al. Value of retrospective fusion of PET and MR images in detection of hepatic metastases: comparison with ${ }^{18} \mathrm{~F}-\mathrm{FDG}$ PET/ CT and Gd-EOB-DTPA-enhanced MRI. J Nucl Med. 2010;51:692-699.

67. Schreiter NF, Nogami M, Steffen I, et al. Evaluation of the potential of PET-MRI fusion for detection of liver metastases in patients with neuroendocrine tumours. Eur Radiol. 2012;22:458-467.
68. Buchmann I, Henze M, Engelbrecht S, et al. Comparison of ${ }^{68} \mathrm{Ga}$-DOTATOC PET and ${ }^{111}$ In-DTPAOC (OctreoScan) SPECT in patients with neuroendocrine tumours. Eur J Nucl Med Mol Imaging. 2007;34:1617-1626.

69. Kam MH, Wong DC, Siu S, Stevenson AR, Lai J, Phillips GE. Comparison of magnetic resonance imaging-fluorodeoxy-glucose positron emission tomography fusion with pathological staging in rectal cancer. Br J Surg. 2010;97:266-268.

70. Al-Sukhni E, Milot L, Fruitman M, et al. Diagnostic accuracy of MRI for assessment of $\mathrm{T}$ category, lymph node metastases, and circumferential resection margin involvement in patients with rectal cancer: a systematic review and metaanalysis. Ann Surg Oncol. January 20, 2012 [Epub ahead of print].

71. Muthusamy VR, Chang KJ. Optimal methods for staging rectal cancer. Clin Cancer Res. 2007; 13:6877s-6884s.

72. Grassetto G, Marzola MC, Minicozzi A, Al-Nahhas A, Rubello D. F-18 FDG PET/CT in rectal carcinoma: where are we now? Clin Nucl Med. 2011;36:884888.

73. Kim DJ, Kim JH, Ryu YH, Jeon TJ, Yu JS, Chung JJ. Nodal staging of rectal cancer: high-resolution pelvic MRI versus ${ }^{18} \mathrm{~F}-\mathrm{FDGPET} / \mathrm{CT}$. J Comput Assist Tomogr. 2011;35:531-534.

74. Wieder HA, Rosenberg R, Lordick F, et al. Rectal cancer: MR imaging before neoadjuvant chemotherapy and radiation therapy for prediction of tumor-free circumferential resection margins and long-term survival. Radiology. 2007;243:744-751.

75. Park MJ, Kim SH, Lee SJ, Jang KM, Rhim H. Locally advanced rectal cancer: added value of diffusion-weighted MR imaging for predicting tumor clearance of the mesorectal fascia after neoadjuvant chemotherapy and radiation therapy. Radiology. 2011;260:771-780.

76. Curvo-Semedo L, Lambregts DM, Maas M, Beets GL, Caseiro-Alves F, BeetsTan RG. Diffusion-weighted MRI in rectal cancer: apparent diffusion coefficient as a potential noninvasive marker of tumor aggressiveness. J Magn Reson Imaging. January 23, 2012 [Epub ahead of print].

77. Lambregts DM, Cappendijk VC, Maas M, Beets GL, Beets-Tan RG. Value of MRI and diffusion-weighted MRI for the diagnosis of locally recurrent rectal cancer. Eur Radiol. 2011;21:1250-1258.

78. Vliegen RF, Beets GL, Lammering G, et al. Mesorectal fascia invasion after neoadjuvant chemotherapy and radiation therapy for locally advanced rectal cancer: accuracy of MR imaging for prediction. Radiology. 2008;246:454-462.

79. Vliegen RF, Beets-Tan RG, Vanhauten B, et al. Can an FDG-PET/CT predict tumor clearance of the mesorectal fascia after preoperative chemoradiation of locally advanced rectal cancer? Strahlenther Onkol. 2008;184:457-464.

80. Moore HG, Akhurst T, Larson SM, Minsky BD, Mazumdar M, Guillem JG. A case-controlled study of 18-fluorodeoxyglucose positron emission tomography in the detection of pelvic recurrence in previously irradiated rectal cancer patients. J Am Coll Surg. 2003;197:22-28. 